\title{
DISTRIBUTION FUNCTIONS FOR THE TIME-AVERAGED ENERGIES OF STOCHASTICALLY EXCITED SOLAR $p$-MODES
}

\author{
Pawan Kumar, Joel Franklin, and Peter Goldreich \\ California Institute of Technology \\ Received 1987 August 12; accepted 1987 November 9
}

\begin{abstract}
We study the excitation of a damped harmonic oscillator by a random force as a model for the stochastic excitation of a solar $p$-mode by turbulent convection. An extended sequence of observations is required to separate different $p$-modes and thus determine the energies of individual modes. Therefore, the observations yield time-averaged values of the energy. We apply the theory of random differential equations to calculate distribution functions for the time-averaged energy of the oscillator. The instantaneous energy satisfies a Boltzmann distribution. With increasing averaging time the distribution function narrows, and its peak shifts toward the mean energy. We also perform numerical integrations to generate finite sequences of time-averaged energies. These are treated as simulated data from which we obtain approximate probability distributions for the time-averaged energy. A comparison of our calculated distributions with those determined observationally should help to resolve whether the solar $p$-modes are stochastically excited. If they are, modes of the same frequency with degree $l \lesssim 200$ should have identical values for the products of their mean energies, line-widths, and masses. If, in addition, turbulence or radiative dissipation provides the principal damping mechanism, the mean energies should be independent of angular order, $l$.

Subject headings: convection - Sun: interior - Sun: oscillations - turbulence
\end{abstract}

\section{INTRODUCTION}

The frequency, line width, and photospheric velocity amplitude are the primary observables associated with each $p$-mode. The energy in a mode is proportional to the square of its velocity amplitude. If the mode is stochastically excited, its energy, $E$, will fluctuate on the damping time scale. Unfortunately, observations of solar $p$-modes cannot determine the instantaneous energy, $E$, but only, $E_{T}$, the energy averaged over some interval of time, say $-T \leq t \leq T$.

A damped harmonic oscillator excited by a random force provides a simple model for the stochastic excitation of a solar $p$-mode by turbulent convection (Goldreich and Keeley 1977; Goldreich and Kumar 1988). The current investigation is devoted to calculating distributions for $E_{T}$ appropriate to the model harmonic oscillator. It is obvious that the distribution function is a decaying exponential, or Boltzmann, distribution for $T=0$ and that it approaches a $\delta$-function at the mean energy as $T \rightarrow \infty$. We determine distribution functions appropriate to arbitrary averaging times by applying the theory of random differential equations. In addition, we provide examples of approximate distribution functions obtained from finite strings of numerically simulated data. The latter are intended as a guide for interpreting observationally determined distribution functions of $E_{T}$ for solar $p$-modes.

The organization of this paper is as follows. In $\S$ II we describe the model harmonic oscillator and outline the procedure for calculating distribution functions for its time-averaged energy. We develop the method used to compute the eigenvalues required to determine the distribution functions in § III. In § IV we present our theoretical distribution functions. We solve the harmonic oscillator equation numerically in $\S \mathrm{V}$ to generate finite sequences of values for $E_{T}$ which are treated as simulated data to determine approximate distribution functions. We briefly discuss some observational implications of our results for the solar $p$-modes in $\S$ VI.

\section{THE STOCHASTICALLY EXCITED OSCILLATOR}

The differential equation governing the time evolution of the coordinate, $q$, of a damped harmonic oscillator of frequency, $\omega_{0}$, which interacts with a random force, $F(t)$, reads

$$
\frac{d^{2} q}{d t^{2}}+2 \Gamma \frac{d q}{d t}+\omega_{0}^{2} q=\frac{F(t)}{M},
$$

where $M$ is the mass and $\Gamma$ is a positive damping constant. We assume that $F(t)$ is a Gaussian random process. This assumption is not very restrictive because the central limit theorem implies that other processes, such as the Poisson process, reduce to a Gaussian process in the limit of large $N$.

The energy, $E$, consists of a kinetic and a potential part;

$$
E=\frac{M}{2}\left[\left(\frac{d q}{d t}\right)^{2}+\omega_{0}^{2} q^{2}\right] .
$$

Our basic strategy is to expand $x_{1} \equiv \omega_{0} q$ and $x_{2} \equiv d q / d t$ in terms of a set of functions which are orthonormal over the time interval $-T \leq t \leq T$. We define $x$ to be the column vector with components $x_{1}$ and $x_{2}$. Then, we have

$$
\boldsymbol{x}(t)=\sum_{n} A_{n} f_{n} \phi_{n}(t) \quad \text { for } \quad-T \leq t \leq T,
$$


where the $\phi_{n}$ are two-component functions which satisfy

$$
\int_{-T}^{T} d t \phi_{m}^{\dagger}(t) \cdot \phi_{n}(t)=\delta_{m, n},
$$

the $f_{n}$ are uncorrelated, random, Gaussian variables with unit variance, and the $A_{n}$ are constants. Equation (3) is an extension of the Karhunen-Loéve expansion (Davenport and Root 1958) to two stochastic processes.

To determine $A_{m} f_{m}$, we take the dot product of both sides of equation (3) by $\phi_{m}^{\dagger}$, then integrate over $-T \leq t \leq T$. Next, we multiply $A_{m} f_{m}$ by $A_{n}^{*} f_{n}^{*}$ and take the expectation value of the resulting expression. ${ }^{2}$ This procedure yields

$$
\left\langle A_{m} A_{n}^{*} f_{m} f_{n}^{*}\right\rangle=\int_{-T}^{T} d t_{1} \int_{-T}^{T} d t_{2}\left[\phi_{m}^{\dagger}\left(t_{1}\right) \cdot\left\langle\boldsymbol{x}\left(t_{1}\right) x^{\dagger}\left(t_{2}\right)\right\rangle \cdot \phi_{n}\left(t_{2}\right)\right] .
$$

Since $\left\langle f_{m} f_{n}^{*}\right\rangle=\delta_{n, m}$, the $\phi_{n}$ must satisfy the integral equation

$$
\int_{-T}^{T} d t_{2} \mathscr{R}\left(t_{1}, t_{2}\right) \cdot \phi_{n}\left(t_{2}\right)=\lambda_{n} \phi_{n}\left(t_{1}\right),
$$

where

$$
\mathscr{R}\left(t_{1}, t_{2}\right)=\left\langle\boldsymbol{x}\left(t_{1}\right) \boldsymbol{x}^{\dagger}\left(t_{2}\right)\right\rangle
$$

is a $2 \times 2$ matrix. Thus, $\phi_{n}$ is an eigenfunction of the integral operator defined by equation (6) and $A_{n}$ is the square root of the corresponding eigenvalue, $\lambda_{n}$. We may now rewrite equation $(3)$ as

$$
x(t)=\sum_{n}\left(\lambda_{n}\right)^{1 / 2} f_{n} \phi_{n}(t)
$$

We note that $\phi_{2}$ is the time derivative of $\phi_{1}$. To prove this we multiply equation (8) by $f_{n}^{*}$ and take the expectation value of both sides. This yields

$$
\left\langle f_{n}^{*} x(t)\right\rangle=\left(\lambda_{n}\right)^{1 / 2} \phi_{n}(t) \quad \text { or } \quad\left(\phi_{n}\right)_{1}=\left\langle f_{n}^{*} x_{1}(t)\right\rangle /\left(\lambda_{n}\right)^{1 / 2} \quad \text { and } \quad\left(\phi_{n}\right)_{2}=\left\langle f_{n}^{*} x_{2}(t)\right\rangle /\left(\lambda_{n}\right)^{1 / 2} .
$$

But by definition $x_{2}=\omega_{0}^{-1} d x_{1} / d t$. Therefore, the above equation implies that $\left(\phi_{n}\right)_{2}=\omega_{0}^{-1} d\left(\phi_{n}\right)_{1} / d t$.

The operator defined by equation (6) is easily shown to be hermitian. Thus, the eigenvalues, $\lambda_{n}$, are real. Furthermore, rewriting equation (5) using $\left|A_{n}\right|^{2}=\lambda_{n}$ yields

$$
\lambda_{n}=\left\langle\left|\int_{-T}^{T} d t_{1} \phi_{n}^{\dagger}\left(t_{1}\right) \cdot x\left(t_{1}\right)\right|^{2}\right\rangle,
$$

which proves that the $\lambda_{n}$ are positive. Because the matrix $\mathscr{R}$ is real, the eigenfunctions can be chosen to be real. Also, since $\boldsymbol{x}$ is real the $f_{n}$ must be real.

To evaluate the average energy, $E_{T}$, we integrate $|x|^{2}$ using equation (8) and the orthonormality property of the $\phi_{n}$;

$$
E_{T} \equiv \frac{1}{2 T} \int_{-T}^{T} d t E=\frac{M}{4 T} \int_{-T}^{T} d t x^{\dagger} \cdot x=\frac{M}{4 T} \sum_{n} \lambda_{n} f_{n}^{2} \equiv \sum_{n} y_{n} .
$$

Since $f_{n}$ is a Gaussian random variable with unit variance and zero mean the probability distribution for $y_{n}, P\left(y_{n}\right)$, is

$$
P\left(y_{n}\right)=\left(\frac{4 T}{\pi M y_{n} \lambda_{n}}\right)^{1 / 2} \exp \left(\frac{-4 T y_{n}}{M \lambda_{n}}\right),
$$

and the characteristic function, $\mathscr{C}_{y_{n}}\left(\omega_{E}\right)$, is

$$
\mathscr{C}_{y_{n}}\left(\omega_{E}\right)=1 /\left(1-i M \omega_{E} \lambda_{n} / 4 T\right)^{1 / 2}
$$

The characteristic function for $E_{T}$ is just the product of the $\mathscr{C}_{y_{n}}\left(\omega_{E}\right)$.

$$
\mathscr{C}_{E_{T}}\left(\omega_{E}\right)=\prod_{n} \frac{1}{\left(1-i M \omega_{E} \lambda_{n} / 4 T\right)^{1 / 2}} .
$$

The inverse Fourier transform of equation (14) gives the probability distribution, $P\left(E_{T}\right)$, for $E_{T}$. Thus, our main task is to determine the eigenvalues of equation (6). We develop a procedure for doing this in the next section.

\section{DETERMINING THE EIGENVALUES}

This section is divided into three parts. In $\S$ III $a$ we calculate the correlation matrix $\mathscr{R}$ by solving equation (1) for $x$. Next, in $\S$ III $b$, we transform the integral equation (6) into a differential equation for $\phi$. Equipped with this we write down the most general form for $\phi$. Finally, in $\S$ III $c$ we substitute the general form for $\phi$ into the integral equation (6) and reduce the problem of finding the eigenvalues to that of finding the roots of an ordinary algebraic equation.

\footnotetext{
${ }^{1}$ A dagger, $\uparrow$, denotes the complex conjugate and transpose of a matrix.

${ }^{2}$ Angular brackets, \langle\rangle , denote an expectation value.
} 


\section{a) Determining The Correlation Matrix}

Throughout this section we take $F(t)$ to be a Gaussian, white noise, random process. The solution of equation (1) is obtained in the terms of the causal Green's function, $G$, for a damped harmonic oscillator.

$$
q(t)=\frac{1}{M} \int_{-\infty}^{t} d t^{\prime} G\left(t, t^{\prime}\right) F\left(t^{\prime}\right),
$$

where

$$
G\left(t, t^{\prime}\right)=\frac{1}{\omega_{1}} \theta\left(t-t^{\prime}\right) \exp \left[-\Gamma\left(t-t^{\prime}\right)\right] \sin \omega_{1}\left(t-t^{\prime}\right) .
$$

Here $\theta(t)$ is the Heavyside function, $\theta(t)=0$ for $t<0$ and $\theta(t)=1$ for $t>0$.

Using equations (15) and (16) we determine the correlation matrix, $\mathscr{R}$, defined by equation (7), to be

$$
\mathscr{R}_{11} \equiv\left\langle x_{1}\left(t_{1}\right) x_{1}\left(t_{2}\right)\right\rangle=\frac{g \omega_{0}}{4 M^{2} \Gamma \omega_{1}} \exp \left(-\Gamma\left|t_{1}-t_{2}\right|\right) \cos \left(\omega_{1}\left|t_{1}-t_{2}\right|-\alpha\right) \equiv \frac{g \omega_{0}}{4 M^{2} \Gamma \omega_{1}} R_{0},
$$

where

$$
\omega_{1}^{2}=\omega_{0}^{2}-\Gamma^{2}, \quad \cos \alpha=\frac{\omega_{1}}{\omega_{0}}, \quad \sin \alpha=\frac{\Gamma}{\omega_{0}} .
$$

In deriving equation (17) we have used $\left\langle F\left(t_{1}\right) F\left(t_{2}\right)\right\rangle=g \delta\left(t_{1}-t_{2}\right)$. The other components of $\mathscr{R}$ may be expressed in terms of $R_{0}$. We find

$$
\mathscr{R}\left(t_{1}, t_{2}\right)=\frac{g \omega_{0}}{4 M^{2} \omega_{1} \Gamma}\left(\begin{array}{cr}
R_{0} & \frac{d R_{0}}{\omega_{0} d t_{2}} \\
-\frac{d R_{0}}{\omega_{0} d t_{2}} & -\frac{d^{2} R_{0}}{\omega_{0}^{2} d t_{2}^{2}}
\end{array}\right)
$$

Using equation (17)

$$
\mathscr{R}\left(t_{1}, t_{2}\right)=\frac{g \omega_{0}}{4 M^{2} \omega_{1} \Gamma} \exp \left(-\Gamma\left|t_{1}-t_{2}\right|\right)\left[\begin{array}{cc}
\cos \left(\omega_{1}\left|t_{1}-t_{2}\right|-\alpha\right) & \sin \omega_{1}\left(t_{1}-t_{2}\right) \\
-\sin \omega_{1}\left(t_{1}-t_{2}\right) & \cos \left(\omega_{1}\left|t_{1}-t_{2}\right|+\alpha\right)
\end{array}\right] .
$$

\section{b) Differential Equation For Eigenvectors}

In order to derive the general form of an eigenvector it is useful to convert the integral equation (6) into a differential equation. To achieve this we exploit a special property of the Fourier transform of the kernel $\mathscr{R}$.

Let

$$
\mathscr{R}(\tau)=\frac{1}{(2 \pi)^{1 / 2}} \int_{-\infty}^{\infty} d \omega \tilde{\mathscr{R}}(\omega) \exp (i \omega \tau),
$$

where $\tau \equiv t_{1}-t_{2}$. Then $\tilde{R}(\omega)$ is determined by taking the Fourier transform of equation (20):

$$
\tilde{\mathscr{R}}(\omega)=\frac{g \omega_{0}^{2}}{(2 \pi)^{1 / 2} M^{2}\left[\omega^{4}+\omega_{0}^{4}-2 \omega^{2} \omega_{1}^{2} \cos (2 \alpha)\right]}\left(\begin{array}{cc}
\frac{-i \omega}{\omega_{0}} \\
\frac{i \omega}{\omega_{0}} & \frac{\omega^{2}}{\omega_{0}^{2}}
\end{array}\right) .
$$

We note that $\tilde{\mathscr{R}}(\omega)$ is the ratio of rational functions. This property enables us to convert the integral equation into a differential equation. Rewriting equation (6) in terms of $\tilde{\mathscr{R}}(\omega)$ we arrive at

$$
\frac{1}{(2 \pi)^{1 / 2}} \int_{-T}^{T} d t^{\prime} \int_{-\infty}^{\infty} d \omega \exp i \omega\left(t-t^{\prime}\right) \frac{\mathscr{P}(i \omega) \cdot \phi\left(t^{\prime}\right)}{Q(i \omega)}=\lambda \phi(t)
$$

where

$$
\tilde{\mathscr{R}}(\omega) \equiv \frac{\mathscr{P}(i \omega)}{Q(i \omega)}, \quad Q(i \omega) \equiv \omega^{4}+\omega_{0}^{4}-2 \omega_{0}^{2} \omega^{2} \cos 2 \alpha,
$$

and $\mathscr{P}(i \omega)$ is a $2 \times 2$ matrix equal to $Q(i \omega) \tilde{R}(\omega)$. Operating on equation $(23)$ by $Q(d / d t)$ we get

$$
\lambda Q\left(\frac{d}{d t}\right) \phi(t)=(2 \pi)^{1 / 2} \mathscr{P}\left(\frac{d}{d t}\right) \cdot \phi(t) .
$$


Then, combining equations (22) and (25) we obtain a pair of differential equations for the components of $\phi$ :

$$
\begin{aligned}
& \lambda^{\prime} Q(D) \phi_{1}=\omega_{0}\left(\omega_{0} \phi_{1}-D \phi_{2}\right), \quad \lambda^{\prime} Q(D) \phi_{2}=\omega_{0} D \phi_{1}-D^{2} \phi_{2}, \\
& \lambda^{\prime}=\frac{\lambda M^{2}}{g}, \quad D \equiv \frac{d}{d t}, \quad Q(D)=D^{4}+2 \omega_{0}^{2} \cos 2 \alpha D^{2}+\omega_{0}^{4} .
\end{aligned}
$$

Substituting $\phi_{2}=D \phi_{1} / \omega_{0}$ into equations (26) yields uncoupled differential equations for $\phi_{1}$ and $\phi_{2}$ :

$$
Q_{1}(D) \phi_{1}=0 \quad \text { and } \quad Q_{1}(D) \phi_{2}=0,
$$

where

$$
Q_{1}(D)=\lambda^{\prime} Q(D)+D^{2}-\omega_{0}^{2} .
$$

We see from equations (28) and (29) that the general form for the eigenfunctions of equation (6) is a sum of four exponentials, each of which satisfies $Q_{1}(D) \exp \beta_{k} t=0$. Furthermore, the $\beta_{k}$ cannot be equal to either $\Gamma \pm i \omega_{1}$ or $-\Gamma \pm i \omega_{1}$ because the four factors of the operator $Q(D)$ are $D \pm \Gamma \pm i \omega_{1}$. Therefore, if $Q(D) \exp \left(\beta_{k} t\right)=0, Q_{1}(D) \exp \left(\beta_{k} t\right)=\left(D^{2}-\omega_{0}^{2}\right) \exp \left(\beta_{k} t\right) \neq 0$, since $\omega_{0}^{2} \neq(\Gamma$ $\left.\pm i \omega_{1}\right)^{2}$.

We conclude this subsection by summarizing the properties of the eigenfunctions.

$$
\begin{aligned}
& \text { 1. } \phi=\left(\begin{array}{c}
\phi_{1} \\
D \phi_{1} / \omega_{0}
\end{array}\right) . \\
& \text { 2. } \phi_{1}=\sum_{k=1}^{4} a_{k} \exp \left(\beta_{k} t\right), \quad \text { where } \quad \beta_{k} \text { is solution of } Q_{1}\left(i \beta_{k}\right)=0 . \\
& \text { 3. } \beta_{k} \neq \Gamma \pm i \omega_{1} \quad \text { and } \beta_{k} \neq-\Gamma \pm i \omega_{1} .
\end{aligned}
$$

\section{c) Algebraic Equation For Eigenvalues}

We substitute the general form for $\phi$ given by equation (30) into the integral eigenvalue equation (6). Equating the coefficients of the exponentials on both sides of the resulting equation yields a set of algebraic equations which is solved to give the eigenvalues.

The evaluation of the left side of equation (6) is simplified by noting that all of the integrals there can be expressed in terms of the integral of $R_{0} \exp (\beta t)$ which is given below:

$$
\begin{aligned}
2 \int_{-T}^{T} d t^{\prime} R_{0}\left(t, t^{\prime}\right) \exp \left(\beta t^{\prime}\right)= & \frac{8 \Gamma \omega_{0} \omega_{1} \exp (\beta t)}{\left(\beta^{2}+\omega_{0}^{2}\right)^{2}-4 \Gamma^{2} \beta^{2}}-\frac{\exp \left[(t+T)\left(i \omega_{1}-\Gamma\right)-\beta T-i \alpha\right]}{\Gamma+\beta-i \omega_{1}} \\
& -\frac{\exp \left[(T-t)\left(i \omega_{1}-\Gamma\right)+\beta T-i \alpha\right]}{\Gamma-\beta-i \omega_{1}}-\frac{\exp \left[-(t+T)\left(i \omega_{1}+\Gamma\right)-\beta T+i \alpha\right]}{\Gamma+\beta+i \omega_{1}} \\
& -\frac{\exp \left[(t-T)\left(i \omega_{1}+\Gamma\right)+\beta T+i \alpha\right]}{\Gamma-\beta+i \omega_{1}} .
\end{aligned}
$$

The denominators in this equation do not vanish because $\beta \neq \Gamma \pm i \omega_{1}$ and $\beta \neq-\Gamma \pm i \omega_{1}$.

Armed with the above expression and equation (19), we are ready to evaluate the integral in equation (6) for the general $\phi$ given by equation (30). The first component of equation (6) yields

$$
\begin{aligned}
2 \int_{-T}^{T} d t^{\prime}\left[R_{0}\left(t, t^{\prime}\right) \phi_{1}\left(t^{\prime}\right)+\frac{1}{\omega_{0}^{2}} \frac{d R_{0}}{d t^{\prime}} \frac{d \phi_{1}}{d t^{\prime}}\right]= & \sum_{k=1}^{4} \frac{8 \Gamma\left(\omega_{0}^{2}-\beta_{k}^{2}\right) a_{k} \exp \left(\beta_{k} t\right) \cos \alpha}{\left(\beta_{k}^{2}+\omega_{0}^{2}\right)^{2}-4 \Gamma^{2} \beta_{k}^{2}} \\
& -\sum_{k=1}^{4} \frac{a_{k}\left[\omega_{0}^{2}-\beta_{k}\left(i \omega_{1}-\Gamma\right)\right]}{\omega_{0}^{2}\left(\Gamma+\beta_{k}-i \omega_{1}\right)} \exp \left[(t+T)\left(i \omega_{1}-\Gamma\right)-\beta_{k} T-i \alpha\right] \\
& -\sum_{k=1}^{4} \frac{a_{k}\left[\omega_{0}^{2}+\beta_{k}\left(i \omega_{1}-\Gamma\right)\right]}{\omega_{0}^{2}\left(\Gamma-\beta_{k}-i \omega_{1}\right)} \exp \left[(T-t)\left(i \omega_{1}-\Gamma\right)+\beta_{k} T-i \alpha\right] \\
& -\sum_{k=1}^{4} \frac{a_{k}\left[\omega_{0}^{2}+\beta_{k}\left(i \omega_{1}+\Gamma\right)\right]}{\omega_{0}^{2}\left(\Gamma+\beta_{k}+i \omega_{1}\right)} \exp \left[-(t+T)\left(i \omega_{1}+\Gamma\right)-\beta_{k} T+i \alpha\right] \\
& -\sum_{k=1}^{4} \frac{a_{k}\left[\omega_{0}^{2}-\beta_{k}\left(i \omega_{1}+\Gamma\right)\right]}{\omega_{0}^{2}\left(\Gamma-\beta_{k}+i \omega_{1}\right)} \exp \left[(t-T)\left(i \omega_{1}+\Gamma\right)+\beta_{k} T+i \alpha\right] \\
& =\frac{8 \Gamma \omega_{1} \lambda^{\prime}}{\omega_{0}} \sum_{k=1}^{4} a_{k} \exp \left(\beta_{k} t\right)
\end{aligned}
$$


Comparing the coefficients of the exponential functions, $\exp \beta_{k} t$, on the two sides of the last equation, and recalling that cos $\alpha=$ $\omega_{1} / \omega_{0}$, we find

$$
\lambda^{\prime}=\frac{\omega_{0}^{2}-\beta_{k}^{2}}{\left(\beta_{k}^{2}+\omega_{0}^{2}\right)^{2}-4 \Gamma^{2} \beta_{k}^{2}} \quad \text { for } \quad k=1,2,3,4 .
$$

Furthermore, the coefficients of $\exp t\left( \pm \pm i \omega_{1}\right)$ must be zero because $\beta_{k} \neq \Gamma \pm i \omega_{1}$ and $\beta_{k} \neq-\Gamma \pm i \omega_{1}$. This yields four equations of constraint on the $a_{k}$ :

$$
\begin{array}{lll}
\sum_{k=1}^{4} a_{k}\left(\frac{\Gamma+\beta_{k}+i \omega_{1}}{\Gamma+\beta_{k}-i \omega_{1}}\right) \exp \left(-\beta_{k} T\right)=0, & \sum_{k=1}^{4} a_{k}\left(\frac{\Gamma-\beta_{k}+i \omega_{1}}{\Gamma-\beta_{k}-i \omega_{1}}\right) \exp \left(+\beta_{k} T\right)=0, \\
\sum_{k=1}^{4} a_{k}\left(\frac{\Gamma+\beta_{k}-i \omega_{1}}{\Gamma+\beta_{k}+i \omega_{1}}\right) \exp \left(-\beta_{k} T\right)=0, & \sum_{k=1}^{4} a_{k}\left(\frac{\Gamma-\beta_{k}-i \omega_{1}}{\Gamma-\beta_{k}+i \omega_{1}}\right) \exp \left(+\beta_{k} T\right)=0
\end{array}
$$

The existence of a nonzero solution for the $a_{k}$ requires that the determinant of the $4 \times 4$ matrix of the coefficients given by equations (34) vanish. This condition gives one equation for the $\beta_{k}$. Equations (33) provide three more independent constraints on the $\beta_{k}$. These four equations can be solved simultaneously to yield the four $\beta_{k}$. Next, we reduce the four $\beta_{k}$ to a single unknown and also reduce the $4 \times 4$ matrix equation to $2 \times 2$ block diagnonal form.

Consider two of the four $\beta_{k}$, say $\beta_{1}$ and $\beta_{2}$, such that $\beta_{1}^{2} \neq \beta_{2}^{2}$. Equation (33) is solved to provide a relation between $\beta_{1}$ and $\beta_{2}$,

$$
\beta_{1}^{2}+\beta_{2}^{2}=4 \Gamma^{2}-2 \omega_{0}^{2}-1 / \lambda^{\prime} \text {. }
$$

It is easy to see from equation (35) that $\beta_{2}$, and hence each of the $\beta_{k}$, must be either real or imaginary. We have shown in $\S$ II that the $\lambda$ are positive. It follows that if $\Gamma<\omega_{0} / 2$ equation (35) implies that at least one of the $\beta_{k}$ must be imaginary. We name the imaginary $\beta_{k}, \beta_{1}$. Since $Q$ is an even polynomial, the $\beta_{k}$ must come in pairs of opposite sign. Thus, without loss of generality, the four $\beta_{k}$ may be taken to be $\beta_{1}, \beta_{2},-\beta_{1}$ and $-\beta_{2}$

The first and second constraint equations (34) now take the form

$$
\begin{aligned}
& \sum_{k=1}^{2}\left[a_{k}\left(\frac{\Omega_{+}+\beta_{k}}{\Omega_{-}+\beta_{k}}\right) \exp \left(-\beta_{k} T\right)+b_{k}\left(\frac{\Omega_{+}-\beta_{k}}{\Omega_{-}-\beta_{k}}\right) \exp \left(+\beta_{k} T\right)\right]=0, \\
& \sum_{k=1}^{2}\left[a_{k}\left(\frac{\Omega_{+}-\beta_{k}}{\Omega_{-}-\beta_{k}}\right) \exp \left(+\beta_{k} T\right)+b_{k}\left(\frac{\Omega_{+}+\beta_{k}}{\Omega_{-}+\beta_{k}}\right) \exp \left(-\beta_{k} T\right)\right]=0,
\end{aligned}
$$

where $\Omega_{ \pm}=\Gamma \pm i \omega_{1}, b_{1}=a_{3}$ and $b_{2}=a_{4}$. Adding and subtracting these two equations, we obtain

$$
\begin{aligned}
& \sum_{k=1}^{2} z_{k}\left[\left(\frac{\Omega_{+}+\beta_{k}}{\Omega_{-}+\beta_{k}}\right) \exp \left(-\beta_{k} T\right)+\left(\frac{\Omega_{+}-\beta_{k}}{\Omega_{-}-\beta_{k}}\right) \exp \left(\beta_{k} T\right)\right]=0, \\
& \sum_{k=1}^{2} z_{k}^{\prime}\left[\left(\frac{\Omega_{+}+\beta_{k}}{\Omega_{-}+\beta_{k}}\right) \exp \left(-\beta_{k} T\right)-\left(\frac{\Omega_{+}-\beta_{k}}{\Omega_{-}-\beta_{k}}\right) \exp \left(\beta_{k} T\right)\right]=0,
\end{aligned}
$$

where

$$
z_{1}=a_{1}+b_{1}, \quad z_{2}=a_{2}+b_{2}, \quad z_{1}^{\prime}=a_{1}-b_{1}, \quad z_{2}^{\prime}=a_{2}-b_{2} .
$$

Two other equations in the $z_{k}$ and the $z_{k}^{\prime}$ are obtained by interchanging $\Omega_{+}$and $\Omega_{-}$in equation (37).

The advantage of these new variables over the $a_{k}$ is that they reduce the $4 \times 4$ matrix which expresses the equations of constraint to $2 \times 2$ block diagonal form. For convenience, we define $\beta_{j}^{(1)}=\beta_{j}$ and $\beta_{j}^{(2)}=-\beta_{j}$ for $j=1$, 2 . Then, the requirement that the determinant of the $4 \times 4$ matrix vanish reduces to the vanishing of the determinant of either one of the following $2 \times 2$ matrices:

$$
\left|\begin{array}{ll}
\sum_{k=1}^{2}\left(\frac{\Omega_{+}+\beta_{1}^{(k)}}{\Omega_{-}+\beta_{1}^{(k)}}\right) \exp \left(-\beta_{1}^{(k)} T\right) ; & \sum_{k=1}^{2}\left(\frac{\Omega_{+}+\beta_{2}^{(k)}}{\Omega_{-}+\beta_{2}^{(k)}}\right) \exp \left(-\beta_{2}^{(k)} T\right) \\
\sum_{k=1}^{2}\left(\frac{\Omega_{-}+\beta_{1}^{(k)}}{\Omega_{+}+\beta_{1}^{(k)}}\right) \exp \left(-\beta_{1}^{(k)} T\right) ; & \sum_{k=1}^{2}\left(\frac{\Omega_{-}+\beta_{2}^{(k)}}{\Omega_{+}+\beta_{2}^{(k)}}\right) \exp \left(-\beta_{2}^{(k)} T\right)
\end{array}\right|=0,
$$

or

$$
\left|\begin{array}{ll}
\sum_{k=1}^{2} i^{2 k}\left(\frac{\Omega_{+}+\beta_{1}^{(k)}}{\Omega_{-}+\beta_{1}^{(k)}}\right) \exp \left(-\beta_{1}^{(k)} T\right) ; & \sum_{k=1}^{2} i^{2 k}\left(\frac{\Omega_{+}+\beta_{2}^{(k)}}{\Omega_{-}+\beta_{2}^{(k)}}\right) \exp \left(-\beta_{2}^{(k)} T\right) \\
\sum_{k=1}^{2} i^{2 k}\left(\frac{\Omega_{-}+\beta_{1}^{(k)}}{\Omega_{+}+\beta_{1}^{(k)}}\right) \exp \left(-\beta_{1}^{(k)} T\right) ; & \sum_{k=1}^{2} i^{2 k}\left(\frac{\Omega_{-}+\beta_{2}^{(k)}}{\Omega_{+}+\beta_{2}^{(k)}}\right) \exp \left(-\beta_{2}^{(k)} T\right)
\end{array}\right|=0 .
$$

Here, $\beta_{2}$ is determined in terms of $\beta_{1}$ from equation (35). This completes our reduction of the integral eigenvalue equation (6) to an algebraic equation. 


\section{THE DISTRIBUTION FUNCTIONS}

We solve the independent transcendental equations (39) and (40) numerically to obtain two infinite sequences of values for $\beta_{1}$. Given $\beta_{1}$ the eignevalue $\lambda^{\prime}$ is obtained from equation (33). We arrange the eigenvalues in each sequence in order of decreasing size and label them by consecutive positive and negative integers, respectively. The sum of all the eigenvalues, $\lambda_{n}^{\prime}$, is equal to $T / \Gamma$. This result follows from equating the expectation value of the integral over $(-T, T)$ of the absolute square of equation (8) to $2 T$ times the trace of $\mathscr{R}(t, t)$ obtained from equation (20). The largest eigenvalues, divided by $T / \Gamma$, are displayed in Figure 1 for several values of $\Gamma T$. Note that the width of the peak decreases with increasing $\Gamma T$ and that there is approximately unit area under each curve.

To obtain an accurate expression for the characteristic function $\mathscr{C}_{E_{T}}$, the sum of the eigenvalues used in equation (14) must be close to $T / \Gamma$. As equation (33) indicates, $\lambda\left(\beta_{1}\right)$ peaks for $\beta_{1}$ close to $i \omega_{0}$ and is independent of $T$. In our determinations of $\mathscr{C}_{E_{T}}$ we use only the few largest $\lambda_{n}$ for $\Gamma T \lesssim 1$ but more than a hundred for $\Gamma T \gtrsim 10$.

The probability distribution function is obtained by taking the inverse Fourier transform of $\mathscr{C}_{E_{T}}$. It is a function of twodimensionless parameters, $\Gamma T$ and $\Gamma / \omega_{0}$. For $\Gamma / \omega_{0} \ll 1$, the case of interest to us, the dependence on $\Gamma / \omega_{0}$ is very weak and may be ignored. Figure 2 displays the distribution function for several values of $\Gamma T$. For $\Gamma T \ll 1$ the distribution function has the Boltzmann form; that is, it is a decreasing exponential. With increasing $\Gamma T$ the peak of the distribution functions narrows and it shifts toward the mean energy of the oscillator. For $\Gamma T \gg 1$ the distribution function tends to a $\delta$-function. The limiting distributions can be obtained analytically from equations (6), (19), and (30).

Our calculations for the distribution functions successfully passed the following three tests. Both the integrated distribution functions and the mean energy are independent of $T$ and the sum of the $\lambda_{n}^{\prime}$ are equal to $T / \Gamma$. There is one additional test of our calculations. For $\Gamma T \gtrsim 1$ the orthogonal series expansion of $\boldsymbol{x}$ is approximately the same as the Fourier series expansion over the interval $(-T, T)$, as expected.

\section{NUMERICAL SIMULATIONS}

We can model the random force $F(t)$ as a Poisson process by setting it equal to a random sequence of $\delta$-function impulses which occur at times $t_{n}$. The time intervals

$$
\Delta t_{n} \equiv t_{n+1}-t_{n}
$$

are independent random variables. The probability density for the waiting time between impulses, $\Delta t$, is given by

$$
P(\Delta t)=r \exp (-r \Delta t)
$$

where $r$ denotes the average number of impulses per unit time. The random variable $\Delta t$ may be generated from a random variable $y$ which is uniformly distributed on the interval $(0,1)$ by using the relation

$$
\Delta t=-\frac{1}{r} \ln (y)
$$

Between impulses $q(t)$ satisfies the homogeneous version of equation (1) and can be written in the form

$$
q_{n}(t)=A_{n} \exp \left[-\left(\Gamma-i \omega_{1}\right)\left(t-t_{n}\right)\right]+A_{n}^{*} \exp \left[-\left(\Gamma+i \omega_{1}\right)\left(t-t_{n}\right)\right],
$$

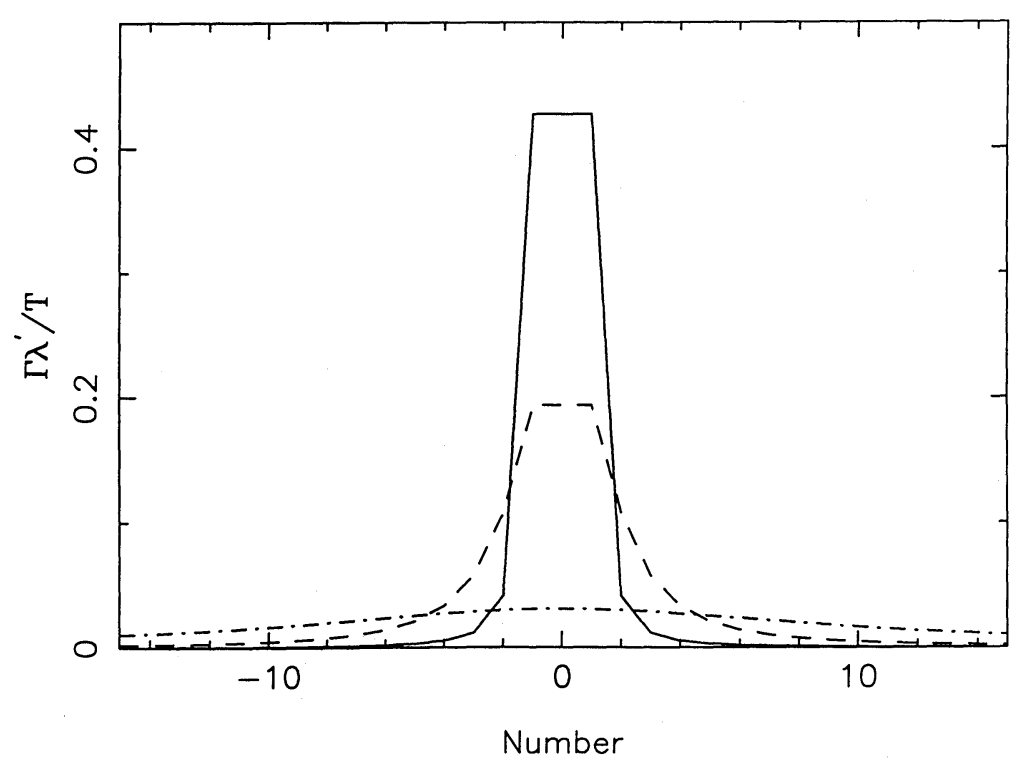

FIG. 1.-Plot of the largest eigenvalues $\left(\lambda^{\prime}\right)$ times $\Gamma / T$ for the two families of eigenfunctions for three cases of averaging times. The eigenvalues have been arranged in a descending series and labeled by positive and negative integers for the two families. Solid curve is for $\Gamma T=0.5$, dashed curve is for $\Gamma T=4$, and the dash-dot curve is for $\Gamma T=32$. 
for $t_{n} \leq t<t_{n+1}$. Inserting $F(t)=p \delta\left(t-t_{n+1}\right)$, where $p$ is a random sign ( \pm 1$)$, into equation (15) we obtain

$$
q_{n+1}(t)=q_{n}(t)+\frac{p}{M} G\left(t, t_{n+1}\right) .
$$

Substituting for the Green's function, $G$, from equation (16), equations (44) and (45) define a mapping from $A_{n}$ and $A_{n+1}$, namely,

$$
A_{n+1}=A_{n} \exp \left[-\Delta t_{n}\left(\Gamma-i \omega_{1}\right)\right]-\frac{i p}{2 \omega_{1}} .
$$

We define $E_{n}$ to be the energy immediately following the $n$th impulse at $t=t_{n}$. With the aid of equations (2) and (44) it may be expressed as

$$
E_{n}=M \omega_{0}^{2}\left[4\left|A_{n}\right|^{2}+A_{n}^{2}+A_{n}^{* 2}-A_{n}^{2} \exp (2 i \alpha)-A_{n}^{* 2} \exp (-2 i \alpha)\right]
$$

where $\sin \alpha=\Gamma / \omega_{0}$.

We have now assembled all the ingredients needed to calculate a string of values of $E_{n}$. The initial conditions are specified by choosing a value for $A_{0}$. The time intervals between consecutive impulses are determined from equation (43) with the values of $y$ obtained from a uniform random number generator. The new amplitude and the energy of the oscillator immediately after an impulse are calculated using equations (46) and (47), respectively. Taking a running mean over time $2 T$ of the sequence of values for $E$ yields a sequence of values for $E_{T}$. It is then a straightforward matter to compute approximate distribution functions for $E_{T}$ and to demonstrate empirically that they converge to the distribution functions derived theoretically.

A theoretically computed distribution function for $E_{T}$ corresponds to an infinite data sample, whereas observations inevitably produce a finite data sample. Figure 2 illustrates how observationally determined distribution functions might appear in comparison to our theoretically predicted ones. These plots display approximate distribution functions computed from data simulated for
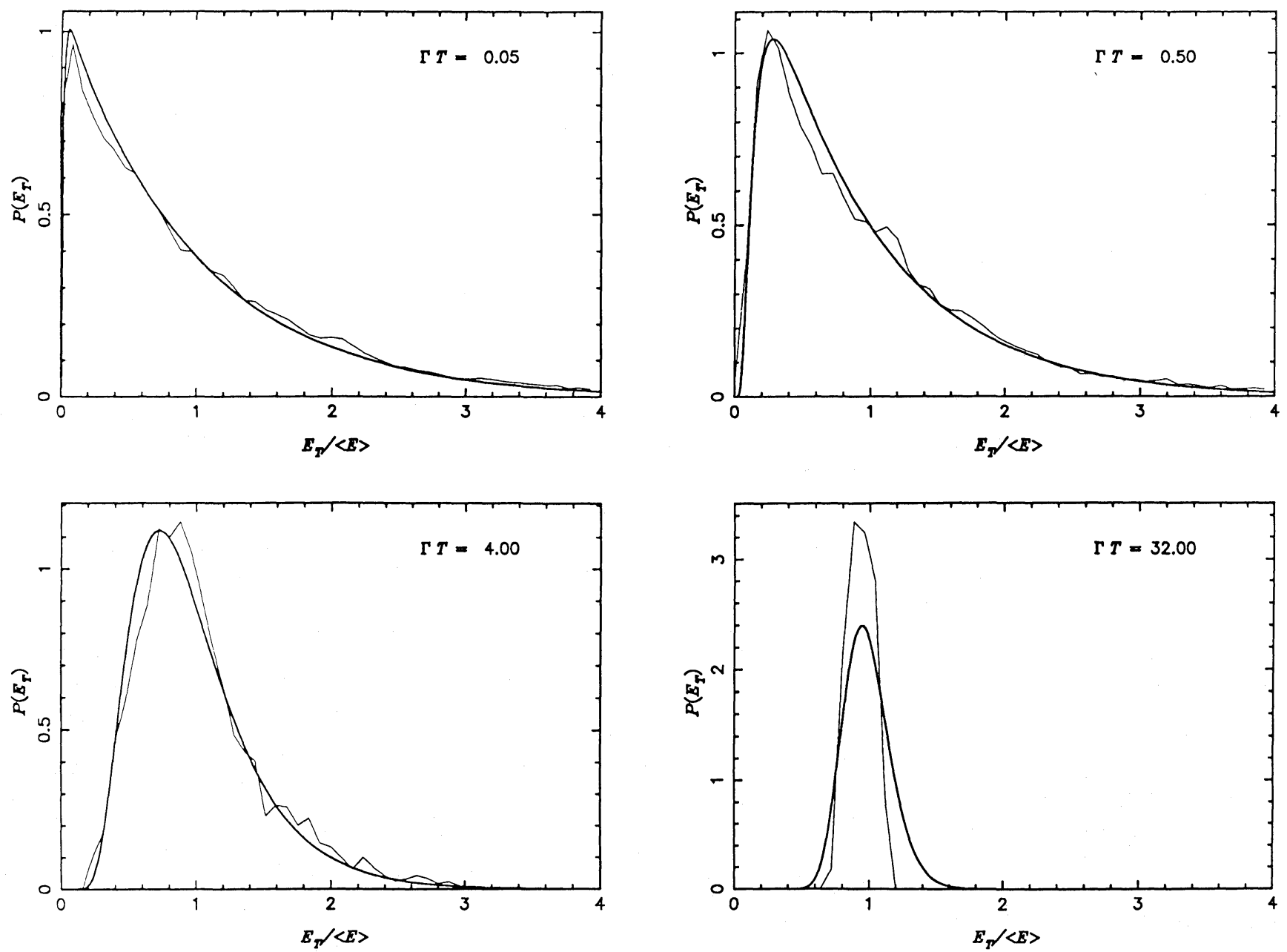

FIG. 2.-The heavy solid curves are theoretically determined distribution functions for four different values of $\Gamma T$, and in each case are same in the range $10 \leq \omega_{0} / \Gamma \leq \infty$. The light solid curves are distribution functions obtained from a string of simulated data 100 daming times long, with $r$ equal to five hits per period and $\omega / \Gamma=10^{3}$. 

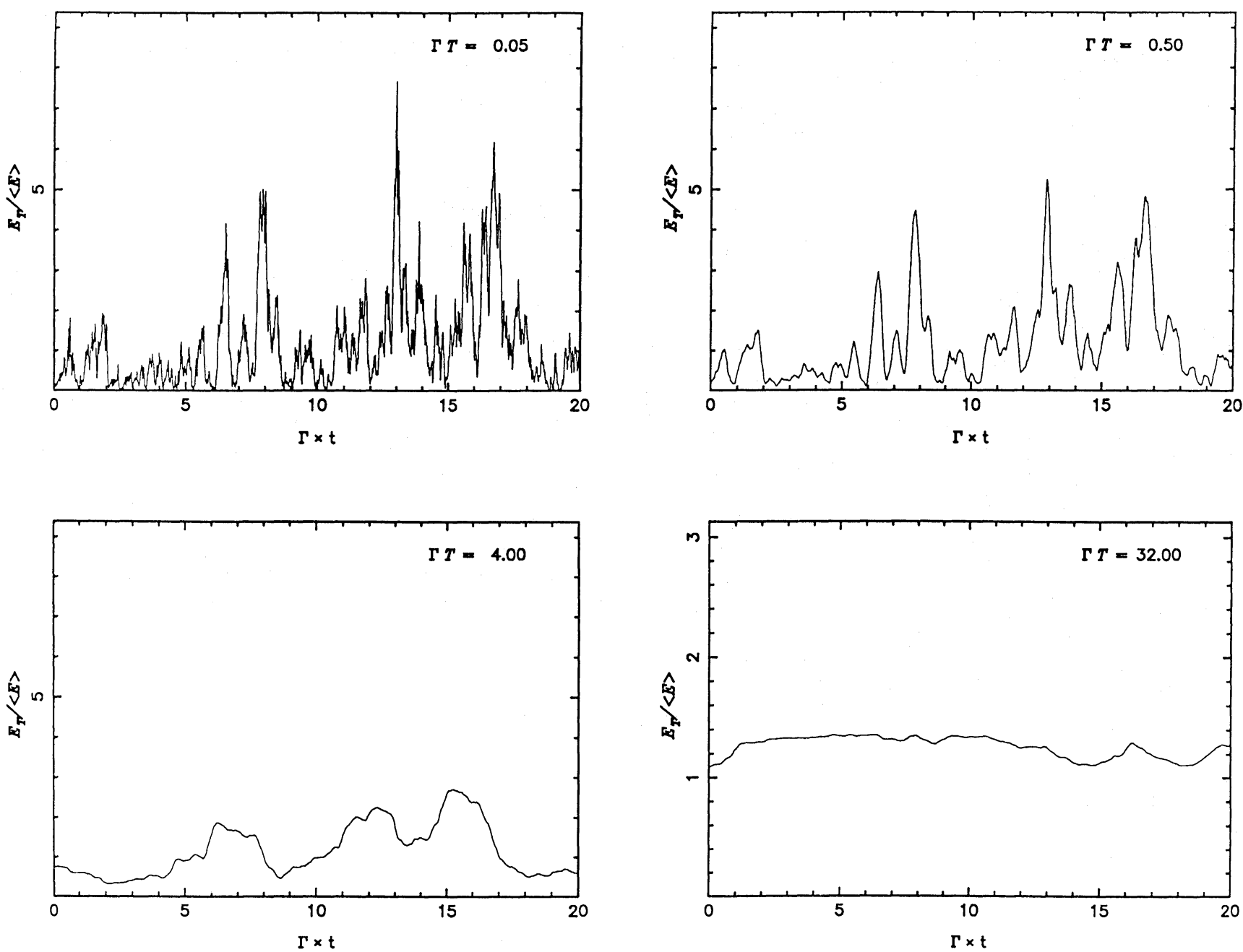

Fig. 3.-A sample of the average energy as a function of time for the four averaging times considered above

100 damping times, along with the theoretical distribution functions. The distribution function obtained from a simulated data of 1000 damping times is quite indistinguishable from the theoretical distribution. In addition, we show in Figure 3 a portion of the strings of values of $E_{T}$ from which each of these distribution functions is obtained.

VI. OBSERVATIONAL IMPLICATIONS

One of our goals is to determine, with the help of the observations, if the solar p-modes are randomly excited. So far our discussion has been rather abstract, and consequently its relevance to the excitation of the solar oscillations may be a bit obscure. Here, we briefly indicate some ways in which the results of this investigation might aid in the understanding of the solar oscillations.

The most obvious application of our results would be to compare the theoretically calculated distribution functions for $E_{T}$ with those determined from observations of solar $p$-modes. The observational determination of the distribution function requires many independent samples of $E_{T}$ for a single mode. Since the lifetimes of the modes in the 5 minute band are of order days, rather lengthy data strings will be required to obtain accurate distribution functions for single modes (see Fig. 2). A more economical approach might be to treat modes with the same $n$ and $l$ but different $m$ as copies of a single mode. It might be still better to further enlarge the set of similar modes to include those with a range of $l$ values at a fixed value of $n$. For instance, lumping together all modes with fixed $n$ having $90<l<110$ and including all values of $m$ gives a set of $\sim 4000$ individual modes.

The spectral density of a random process is the expectation value of the time-averaged, absolute square of the finite Fourier transform in the limit of infinite averaging time. The Wiener-Khinchin theorem (Goodman 1985) states that the spectral density is equal to the Fourier transform of the autocorrelation function. Thus, the spectral density for $q, P_{q}(\omega)$, is equal to $\mathscr{R}_{11}(\omega) / \omega_{0}^{2}$, which from equation (22) is given by

$$
P_{q}(\omega)=\frac{g}{(2 \pi)^{1 / 2} M^{2}} \frac{1}{\left(\omega^{2}-\omega_{0}^{2}\right)^{2}+4 \omega^{2} \Gamma^{2}} .
$$

To a very good approximation $P_{q}(\omega)$ is Lorentzian, with line width, $\Gamma$, near the peak of the power spectrum which is located at 
$\left(\omega_{0}^{2}-2 \Gamma^{2}\right)^{1 / 2}$. Thus, the drag force responsible for the damping produces a small frequency shift,

$$
\Delta \omega \approx-\frac{\Gamma^{2}}{\omega_{0}} .
$$

For solar $p$-modes in the 5 minute band $\Gamma \approx 1 \mu \mathrm{Hz}$ (Libbrecht and Zirin 1986, Isaak 1986). Thus, the frequency shift caused by the decay is $\lesssim 1 \mathrm{nHz}$, which is much smaller than typical errors in the frequency measurements, $\sim 0.1 \mu \mathrm{Hz}$, and is insignificant compared to the discrepancies between the observed and theoretically computed frequencies, $\sim 15 \mu \mathrm{Hz}$.

The mean energy, $\bar{E}$, is equal to $M / 2$ times the trace of $\mathscr{R}(t, t)$. Using equation (20) we obtain

$$
\bar{E}=\frac{g}{4 M \Gamma} \text {. }
$$

We may generalize the expression for $\bar{E}$ so that it applies to an arbitrary random force by replacing $g$ by the spectral density of the random force evaluated at frequency $\omega_{0}$. Thus, different oscillators interacting with random forces which have the same $g\left(\omega_{0}\right)$ should have identical values for the products of their mean energies, line widths, and masses.

The excitation and damping of solar oscillations by turbulent convection are described by effective random forces and damping constants for which $g\left(\omega_{0}\right)$ and $\Gamma$ are given by overlap integrals of particular combinations of modal eigenfunctions and turbulent fields (Goldreich and Keeley 1977; Goldreich and Kumar 1988). Since the excitation and damping by turbulence depend sensitively upon the turbulent Mach number, it is likely that both are dominated by contributions from the upper few scale heights of the solar convection zone.

The radial displacement dominates the horizontal displacement for $p$-modes in the 5 minute band with $l \lesssim 200$. Moreover, the shape of the radial displacement component of the eigenfunction in the upper convection zone depends only on frequency and is independent of $l$. Thus, we would expect modes which have the same frequencies but different values of $l$ and $m$ to have identical values of $M \bar{E} \Gamma$ if they are stochastically excited by turbulent convection. If, in addition, either turbulent convection or radiative dissipation near the photosphere is the major source of damping, $\Gamma$ should be inversely proportional to the mass of the mode. ${ }^{3}$ This would lead to a stronger prediction, namely, that all modes of a given frequency having $l \lesssim 200$ should have the same value of $\bar{E}$.

The observational results of Libbrecht et al. (1986) suggest that the energies of $p$-modes in the 5 minute band are approximately independent of $l$, at least for $l \lesssim 100$.

P. K. is grateful to Ken Libbrecht for many useful discussions. The research reported in this paper was supported by NSF through grant AST-861299.

${ }^{3}$ The mass of a mode is the ratio of its total energy to the square of its rms, photospheric, radial velocity.

\section{REFERENCES}

Davenport, W. B., Jr., and Root, W. L. 1958, An Introduction to the Theory of

Random Signals and Noise (New York: McGraw-Hill).

Goldreich, P., and Keeley, D. 1977, Ap. J., 212, 243.

Goldreich, P., and Kumar, P. 1988, in preparation.

Goodman, J. W. 1985, Statistical Optics (New York: Wiley).

Issak, G. R. 1986, in Seismology of the Sun and the Distant Stars, ed. D. O. Gough (Dordrecht: Reidel.), p. 223.

Libbrecht, K. G., Popp, B. D., Kaufman, J. M., and Penn, M. J. 1986, Nature, 323, 235 .

Libbrecht, K. G., and Zirin, H. 1986, Ap. J., 308, 413.

Joel Franklin and Peter Goldreich: California Institute of Technology, Pasadena, CA 91125

PaWAN Kumar: National Center for Atmospheric Research, High Altitude Observatory, P.O. Box 3000, Boulder, CO $80307-0440$ 